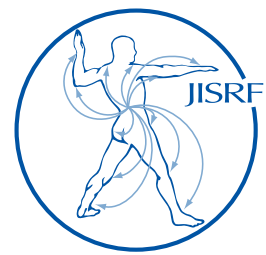

\title{
Medial Tibial Reduction Osteotomy is Associated with Excellent Outcomes and Improved Coronal Alignment
}

\author{
Martin $R^{1}$, Levy $D^{1}$, Miner $T^{1}$, Conrad $D^{2}$, Jennings $J^{1}$, Dennis $D^{1}$
}

\begin{abstract}
Background: The medial tibial reduction osteotomy (MTRO) was introduced to achieve coronal ligamentous balance in total knee arthroplasty (TKA) patients with substantial preoperative varus deformity. Limited data exists on the outcomes of patients requiring an MTRO. This study compares outcomes of a matched cohort of patients that either required or did not require an MTRO during TKA.

Methods: A retrospective review was performed on 67 patients that underwent an MTRO during primary TKA to achieve coronal balance. This patient population was matched 1:1 to another cohort of TKA patients by age, gender, and BMI that did not require an MTRO. A clinical and radiographic evaluation was utilized to compare the two cohorts.

Results: Preoperatively, the tibiofemoral angle was $3.42^{\circ}$ valgus versus $6.12^{\circ}$ varus in the control and MTRO cohorts respectively ( $\mathrm{p}=0.01$ ). Mean postoperative tibiofemoral angles were $3.40^{\circ}$ versus $2.43^{\circ}$ valgus respectively. Postoperative Knee Society Scores were superior in the MTRO cohort (183.84 versus 174.58; $\mathrm{p}=0.04)$. Intraoperatively, no superficial MCL releases were required to achieve coronal balance in either cohort. Complications were similar and limited in both groups. Medial tibial bone resorption was observed in $64 \%$ of MTRO subjects averaging $2.02 \mathrm{~mm}$ versus only $0.3 \mathrm{~mm}$ in the control cohort $(\mathrm{p}=0.01)$.

Conclusion: Patients requiring an MTRO achieved similar alignment and superior knee scores compared to a control cohort with less varus deformity. This procedure eliminated the need for release of the superficial MCL. Resorption of medial tibial bone was commonly observed, possibly secondary to sawinduced thermal necrosis associated with performing an MTRO.
\end{abstract}

Keywords: varus deformity; medial release; total knee replacement; alignment; stability

Level of Evidence: AAOS Therapeutic Level II

1 J. Ryan Martin MD, Daniel Levy BS, Todd M. Miner MD, Jason M. Jennings MD DPT, Douglas A. Dennis MD

Colorado Joint Replacement, 2535 S. Downing Street, Denver, CO USA

2 David Conrad MD

Thedacare Orthopedics, 820 E Grant St, Appleton, WI 54911

(Direct reprint requests to J. Ryan Martin)
(C) 2017 Martin, Levy, Miner, Conrad, Jennings, Dennis. All rights reserved. Authors retain copyright and grant the journal right of first publication with the work. Reconstructive Review follows the $\underline{\text { Creative }}$ Commons Attribution-NonCommercial CC BY-NC. This license

allows anyone to download works, build upon the material, and share them with others for non-commercial purposes as long as they credit the senior author, Reconstructive Review, and the Joint Implant Surgery \& Research Foundation (JISRF). An example credit would be: "Courtesy of (senior author's name), Reconstructive Review, JISRF, Chagrin Falls, Ohio". 


\section{Introduction}

A fixed varus deformity is commonly seen in patients undergoing primary total knee arthroplasty (TKA). The severity of the deformity may lead to longer surgical times, increased difficulty in soft tissue balancing, and trouble achieving optimal bony alignment [1-4]. There are numerous methods of achieving soft tissue balance in the varus knee including a variety of ligament releases, $[\underline{5}, \underline{6}]$ and more recently a medial tibial reduction osteotomy [7-2] (MTRO) has been described. This technique consists of lateralizing the tibial tray with resection of the uncapped medial tibial bone. In some cases, this is associated with downsizing of the tibial tray. The utilization of the MTRO may result in a decrease in operative times when compared to soft tissue releases alone [10] and typically lessens the amount of medial soft tissue releasing required to correct the varus deformity. Additionally, aggressive medial soft tissue release can lead to attenuation of the medial collateral ligament [11] (MCL). The following study describes a treatment algorithm for balancing a fixed varus knee deformity by incorporating a series of medial soft tissue releases and a MTRO to achieve coronal mechanical alignment. Secondary outcomes for this study include: 1) to report what patient or radiographic factors are associated with a need to perform a MTRO, 2) to review the radiographic and clinical outcomes of patients that underwent an MTRO, and 3) to compare the results of patients that required an MTRO to a matched cohort of TKA patients with a varus deformity that did not require a MTRO.

\section{Materials and Methods}

This analysis was initiated after Institutional Review Board approval. A retrospective review of prospectively collected data of patients undergoing primary TKA with varus deformities from 2009 to 2011 at our institution was performed. All patients underwent a primary TKA implanted by the senior author (DAD). All subjects were implanted with a cemented posterior cruciate substituting TKA (PFC Sigma, DePuy, Warsaw, IN). Sixty-seven patients were identified that required an MTRO to achieve coronal balance. This patient population was then matched by age, gender, and BMI to a cohort of 67 TKA patients with preoperative varus deformities that did not require an MTRO during this time period.

\section{Surgical Approach}

Each knee was exposed utilizing a standard medial parapatellar arthrotomy. The distal femoral and proximal tibial resections were initially performed to create the extension gap. Coronal balance was assessed with a spacer block. If the medial extension gap was noted to be tighter than the lateral aspect of the extension gap, a series of steps were performed to achieve a rectangular extension gap (Table 1). A stepwise medial release was performed beginning with releasing the deep medial collateral ligament to the mid-coronal plane of the tibia and removal of femoral and tibial osteophytes which create tension on tightened medial structures. Removal of any distal femoral osteophytes located underneath the superficial medial collateral ligament at this point is particularly important (Figure 1). A spacer block is then inserted into the extension gap to assess gap balance. If medial tightness persists, release of the posteromedial capsule (posterior oblique ligament) was performed. Gap symmetry was reassessed with a spacer block after each release. Occasionally, there were large $(>1 \mathrm{~cm})$ posterior femoral osteophytes that may tent the posteromedial capsule contributing to gap asymmetry. In these cases, a preliminary four millimeter resection of the posterior aspect of the medial femoral condyle was executed with the knee flexed at 90 degrees and a laminar spreader positioned in the lateral aspect of the flexion gap to allow access to the posterior osteophytes (Figure 2A). A curved osteotome was then used to remove the posterior femoral osteophytes (Figure 2B). This step is crucial as these osteophytes can mislead the surgeon into thinking a supporting soft tissue structure is too tight, resulting in a surgical release of that "tight" structure. If the osteophytes are removed late, after soft tissue releases have been performed, unwanted laxity of the released structures is incurred that results in asymmetry of the flexion and extension gaps leading to potential malrotation of the femoral component if a gap balancing surgical technique is utilized.

Table 1. Authors' Sequence Of Steps Utilized To Correct A Varus Deformity And Obtain A Rectangular Extension Gap

1. Release of the deep medial collateral ligament to the midcoronal plane of the tibia

2. Removal of all femoral and tibial osteophytes

3. Release of the posteromedial capsule (posterior oblique ligament)

4. Perform a medial tibial reduction osteotomy

5. Release of the semimembranosus tendon

6. Perforation of the superficial medial collateral ligament with an 18 gauge needle

7. Release of the distal attachment of the superficial medial collateral ligament

If an asymmetric extension gap remains after these sequential techniques, a MTRO was utilized. Initially, the tibial base plate trial was lateralized as much as possible 


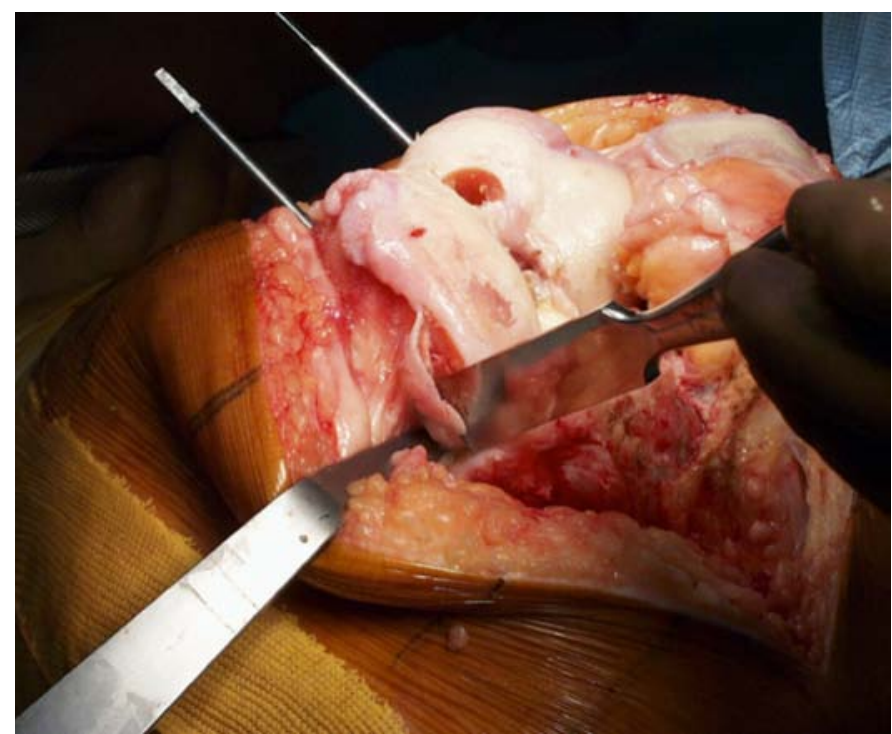

Figure 1. Removal of any distal femoral osteophytes located underneath the superficial medial collateral ligament.

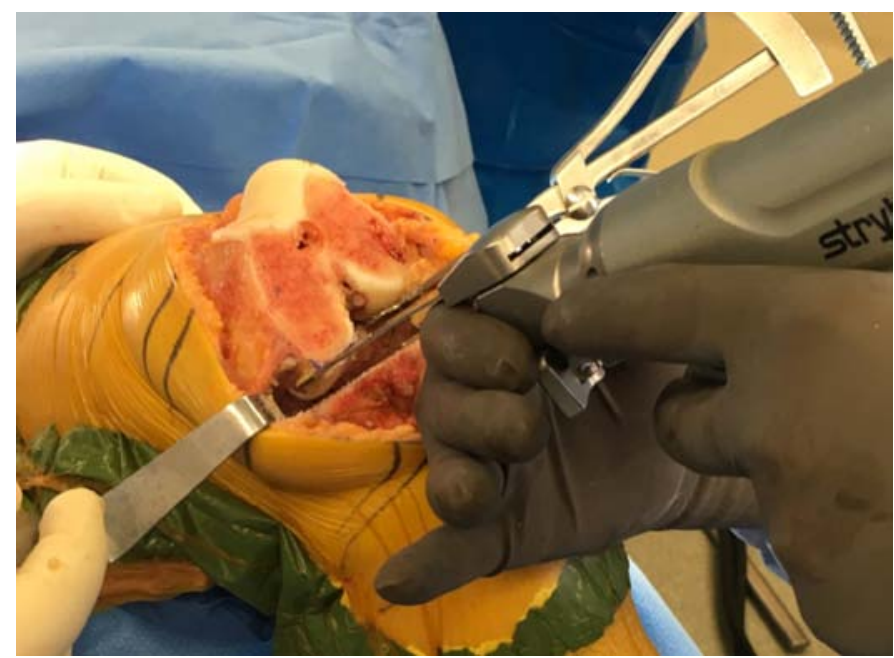

Figure 2A. Four millimeter resection of the posterior aspect of the medial femoral condyle.

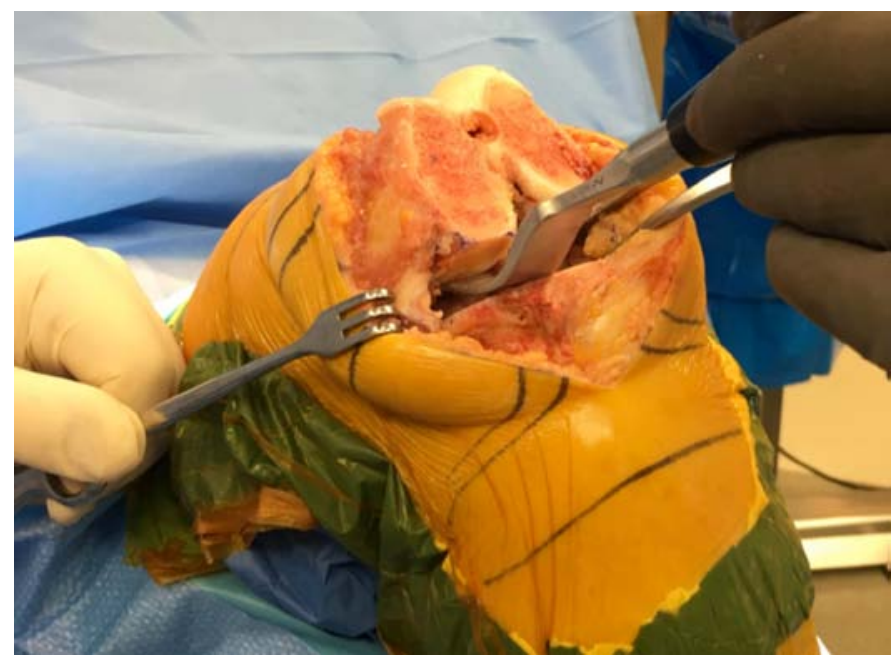

Figure 2B. A curved osteotome was then used to remove the posterior femoral osteophytes.

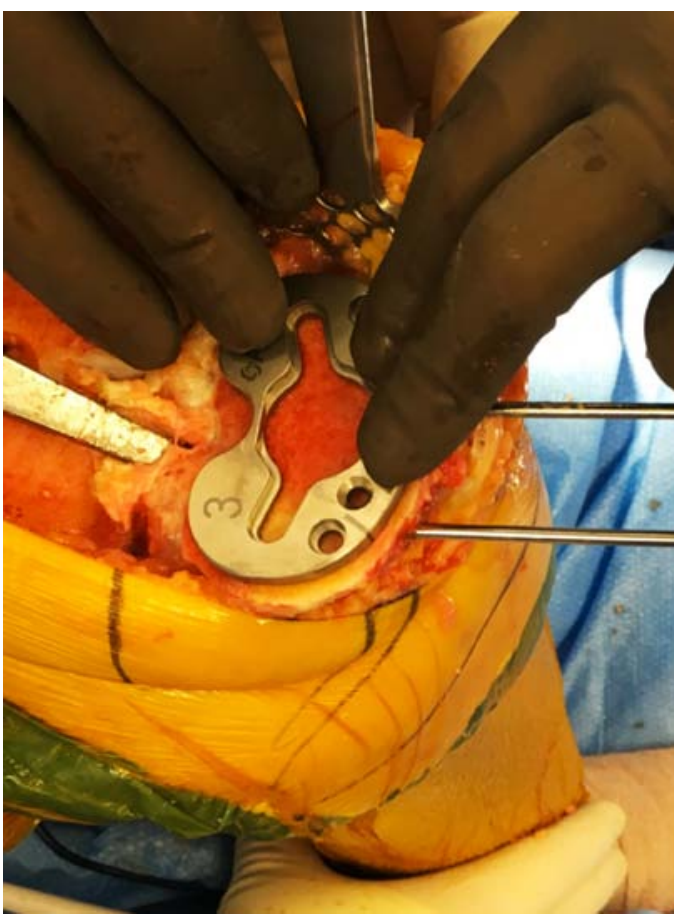

Figure 3A. Initially, the tibial base plate trial was lateralized as much as possible without overhanging the perimeter of the lateral tibial plateau.

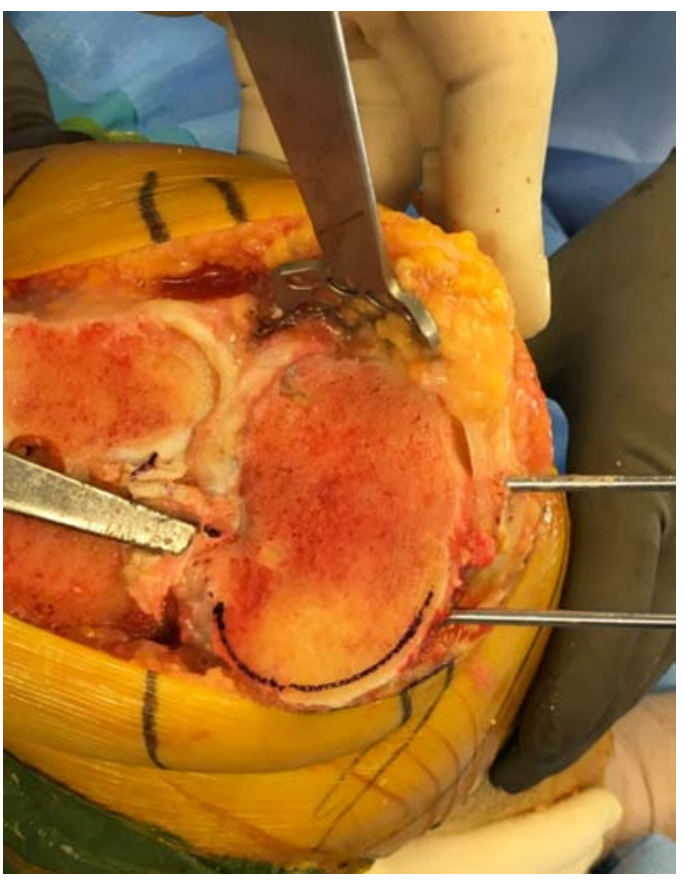

Figure 3B. A marking pen was used to outline the medial extent of the tibial tray on the medial tibial plateau.

without overhanging the perimeter of the lateral tibial plateau (Figure 3A). A marking pen was used to outline the medial extent of the tibial tray on the medial tibial plateau (Figure 3B). The bone that was exposed medial to the outline of the tibial tray was then removed utilizing a combination of an oscillating saw and a rongeur (Figures 4A and $4 \mathrm{~B}$ ). This reduces tension on the medial collateral ligament, facilitating correction of the varus deformity. Lastly, if asymmetry persists after a substantial MTRO was performed ( $>2 \mathrm{~mm}$ difference in gaps), consideration for release of the superficial MCL was considered. 


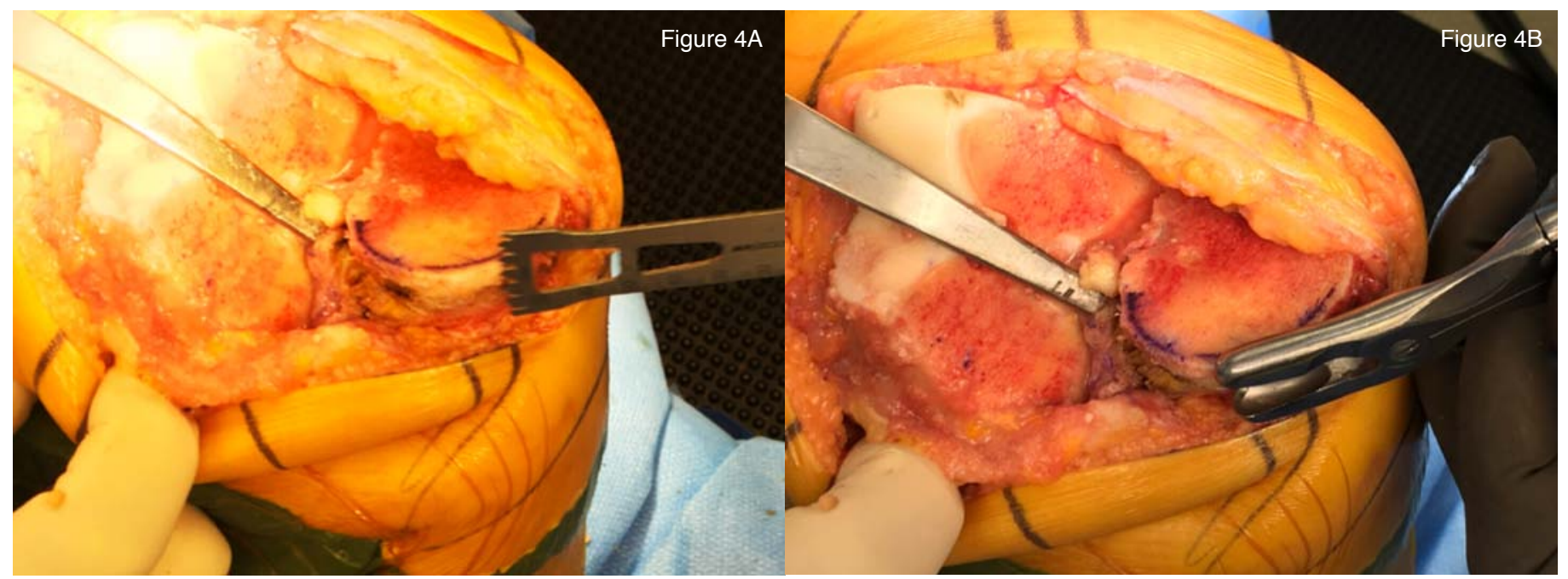

Figure $4 A \& 4 B$. The bone that was exposed medial to the outline of the tibial tray was then removed utilizing a combination of an oscillating saw and a rongeur.

\section{Post-operative protocol}

All patients followed a standardized postoperative rehabilitation protocol, allowing full-weight bearing on the operative extremity immediately after the operative procedure. Patients ambulated with the use of two crutches or a walker for 2 weeks with gradual progression to ambulation without an assistive device over the next 2-4 weeks. Outpatient physical therapy three times weekly for six weeks was utilized for all patients. Postoperative clinic evaluations were performed at 2 weeks, 6 weeks, 3 months, and at 1,2 and 5- years. A functional analysis, physical examination, and radiographic assessment were performed at each follow-up appointment.

\section{Radiographic Analysis}

Preoperative and postoperative radiographs including a standing AP view of both knees, lateral and merchant patellar views, as well as a long leg radiograph were obtained on all subjects. The following data points were analyzed on preoperative films: femoral and tibial articular surface angles and tibiofemoral angle (Figure 5). Six-week fulllength radiographs were reviewed to determine the alignment of the tibial and femoral components, the femoraltibial angle and to assess the status of the medial tibial bone early following MTRO. Final follow-up radiographs were reviewed to determine component position and fixation, overall alignment, and the presence of any medial tibial bone resorption.

\section{Patient Outcomes}

Knee Society scores were recorded at the preoperative and all subsequent postoperative clinical visits. Additionally, all revisions, complications and or reoperations were continually recorded after TKA implantation.

\section{Patient Demographic Data}

We identified 67 patients that underwent an MTRO at the time of TKA. This patient population was then matched to a control cohort of 67 patients with preoperative varus deformity that did not require an MTRO at the time of primary TKA by age, gender, and BMI (Table 2).

The implant data for both cohorts can be seen below in Table 3. Mobile bearings were typically utilized in younger patients and fixed bearing in more senior patients $(>65$ 70 years).

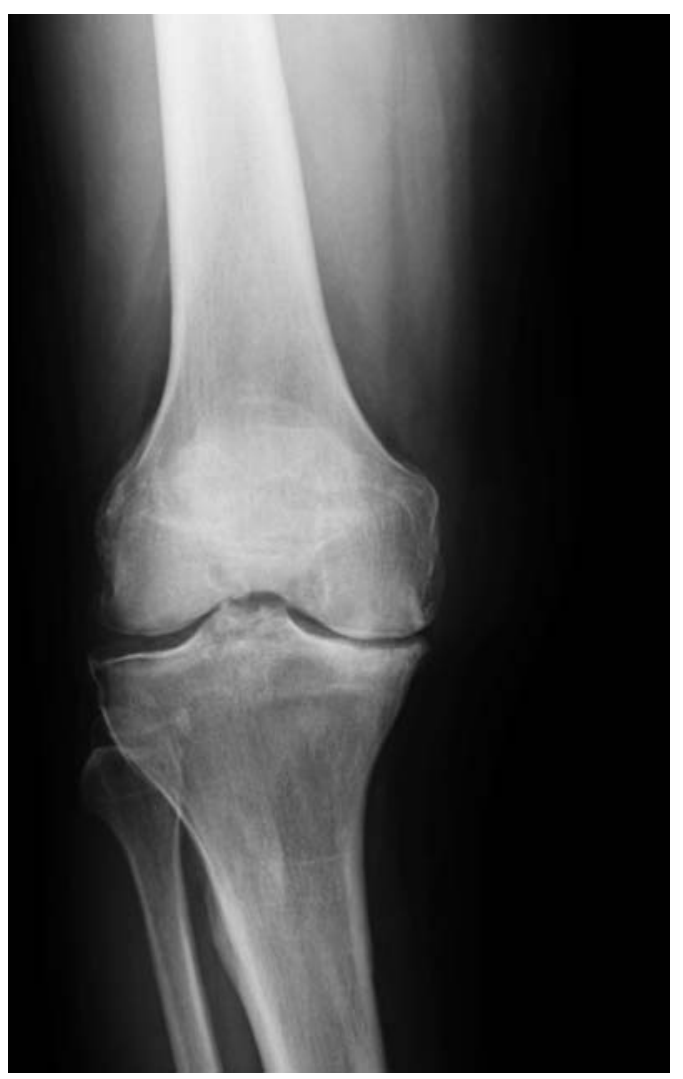

Figure 5. Femoral and tibial articular surface angles and tibiofemoral angle. 
Table 2. Patient Demographics

\begin{tabular}{|l|c|c|c|}
\hline & MTRO Cohort & Control Cohort & P-value \\
\hline Gender & $(13 \mathrm{~F}, 54 \mathrm{M})$ & $(19 \mathrm{~F}, 48 \mathrm{M})$ & \\
\hline Age (years) & $65.81( \pm 7.73)$ & $64.90( \pm 7.87)$ & 0.50 \\
\hline BMI $(\mathrm{kg} / \mathrm{m} 2)$ & $29.49( \pm 4.74)$ & $29.70( \pm 5.30)$ & 0.81 \\
\hline Follow-up (years) & $4.62( \pm 1.14)$ & $4.60( \pm 1.32)$ & 0.91 \\
\hline
\end{tabular}

Table 3. Implant Data

\begin{tabular}{|l|c|c|}
\hline Implant Type & $\begin{array}{c}\text { MTRO } \\
\text { Cohort }\end{array}$ & $\begin{array}{c}\text { Control } \\
\text { Cohort }\end{array}$ \\
\hline PFC Sigma Rotating Platform & 48 & 39 \\
\hline PFC Sigma Fixed Bearing & 7 & 9 \\
\hline PFC Sigma All Polyethylene Tibia & 12 & 18 \\
\hline PFC Sigma TC3 Rotating Platform & 0 & 1 \\
\hline
\end{tabular}

Table 4. Preoperative Radiographic Parameters

\begin{tabular}{|l|c|c|c|}
\hline & MTRO Cohort & Control Cohort & P-value \\
\hline Overall & $86.12( \pm 4.17)$ & $93.42( \pm 7.08)$ & 0.01 \\
\hline Femoral & $96.27( \pm 2.14)$ & $96.99( \pm 2.34)$ & 0.07 \\
\hline Tibial & $85.79( \pm 2.83)$ & $87.54( \pm 2.04)$ & 0.01 \\
\hline
\end{tabular}

Table 5. Postoperative Radiographic Evaluation

\begin{tabular}{|l|c|c|c|}
\hline & MTRO Cohort & Control Cohort & P-value \\
\hline Tibiofemoral angle & $92.43( \pm 1.55)$ & $93.40( \pm 1.62)$ & 0.001 \\
\hline Femoral Angle & $93.69( \pm 1.41)$ & $94.33( \pm 1.72)$ & 0.02 \\
\hline $\begin{array}{l}\text { Tibial Articulation } \\
\text { Surface Angle }\end{array}$ & $89.55( \pm 1.10)$ & $89.00( \pm 0.95)$ & 0.002 \\
\hline $\begin{array}{l}\text { Amount of } \\
\text { Correction }\end{array}$ & $6.31( \pm 4.37)$ & $-0.02( \pm 6.75)$ & 0.01 \\
\hline $\begin{array}{l}\text { Number of Patients } \\
\text { with Medial Tibial } \\
\text { Bone Resorption }\end{array}$ & $43 / 67$ & $2 / 67$ & N/A \\
\hline $\begin{array}{l}\text { Medial Tibial Bone } \\
\text { Resorption }(\mathrm{mm})\end{array}$ & $2.02( \pm 2.18)$ & $0.03( \pm 1.03)$ & 0.01 \\
\hline
\end{tabular}

\section{Statistical Analysis}

Preoperative radiographic image dependent variables analyzed included femoral-tibial angle, femoral component angle, and tibial component angle. Immediate postoperative dependent variables evaluated included the amount of correction (difference between preoperative and postoperative femoral-tibial alignment), overall femoral-tibial angle, femoral component angle, tibial component angle, and the presence of medial tibial bone-cement radiolucent lines. Knee Society scores were considered as dependent variables at preoperative and final follow-up evaluations. Dependent variables were compared across groups using Student's t-test (for equal variances) and Welch's analysis of variance test (for unequal variances). Equality of vari- ance was assessed using Levene's test. A multiple linear regression analysis was used to assess the risk between preoperative radiographs and patient demographics to requiring a MTRO. Pearson product moment correlation was used to assess the correlation between preoperative radiographic results to medial tibial bone resorption at final follow-up evaluation. The level of significance for all inferential statistics was set at (alpha $=0.05)$. All inferential statistics were performed using SPSS v22.0 (IBM Inc.).

\section{Results}

\section{Patient factors associated with increased MTRO}

Utilizing the patient's preoperative radiographs to analyze preoperative tibiofemoral alignment, a higher degree of varus deformity was observed in the MTRO cohort than the control cohort, (3.88 degrees varus vs. 3.42 degrees valgus respectively, $\mathrm{p}=0.01$ ) (Table 4 ). The tibial articular surface angle was aligned in a greater varus posture in the MTRO cohort (85.79 vs. 87.54 degrees, $\mathrm{p}=0.01$ ). There was no statistically significant difference in the femoral articular surface angle between the two groups (96.27 vs. 96.99 degrees, $\mathrm{p}=0.07$ ). No patient in either group required a superficial MCL release to obtain coronal alignment.

\section{Radiographic Outcomes}

The postoperative alignment at final follow-up of the MTRO cohort was statistically significantly different for overall tibiofemoral angle, femoral articular surface angle, and tibial articular surface angle, respectively (Table 5). There was significantly more correction in the MTRO cohort. Medial tibial bone resorption was significantly greater in the MTRO cohort. In the MTRO cohort, $64 \%$ of subjects were noted to have medial tibial bone resorption versus only $3 \%$ in the control cohort $(\mathrm{p}=0.008)$. Additionally, within the MTRO cohort, $77 \%$ had bone resorption with a cobalt chromium tibial tray vs $8 \%$ with an all polyethylene tibial tray $(\mathrm{p}<0.01)$. In the control cohort, $4 \%$ of patients with a cobalt chromium tray had resorption vs. $0 \%$ in the all polyethylene cohort.

\section{Clinical Outcomes}

The preoperative clinical evaluation identified no statistically significant difference in pain scores, extension, flexion, total function, or Knee Society Scores. However, there was a statistically significantly increase in valgus alignment $(\mathrm{p}=0.01)$ and total knee score $(\mathrm{p}=0.004)$ in the control cohort. Postoperatively, there were no statistically significant differences in pain score, extension, flexion, alignment or total Knee Society Scores. There was a statistically 
Table 6: Pre- and Postoperative Clinical Evaluations

\begin{tabular}{|l|c|c|c|}
\hline & MTRO Cohort & Control Cohort & P-value \\
\hline Preoperative & & & \\
\hline Pain Score & $12.38( \pm 10.35)$ & $11.36( \pm 6.84)$ & 0.53 \\
\hline Extension & $7.52( \pm 5.57)$ & $6.67( \pm 6.23)$ & 0.44 \\
\hline Flexion & $119.44( \pm 8.89)$ & $118.96( \pm 9.95)$ & 0.78 \\
\hline Valgus Alignment & $16.71( \pm 7.25)$ & $10.26( \pm 9.11)$ & 0.01 \\
\hline Total Function & $58.57( \pm 15.49)$ & $60.00( \pm 16.50)$ & 0.63 \\
\hline Total Knee & $36.30( \pm 14.89)$ & $43.87( \pm 13.16)$ & 0.004 \\
\hline Total Score & $94.87( \pm 26.02)$ & $103.87( \pm 23.51)$ & 0.05 \\
\hline Final Follow-up & & & \\
\hline Pain Score & $45.00( \pm 9.84)$ & $44.27( \pm 10.11)$ & 0.69 \\
\hline Extension & $0.79( \pm 1.73)$ & $1.84( \pm 4.69)$ & 0.12 \\
\hline Flexion & $124.94( \pm 16.50)$ & $121.93( \pm 14.79)$ & 0.30 \\
\hline Valgus Alignment & $0.32( \pm 2.52)$ & $0.17( \pm 0.91)$ & 0.68 \\
\hline Total Function & $90.00( \pm 14.59)$ & $82.27( \pm 23.07)$ & 0.04 \\
\hline Total Knee & $93.84( \pm 10.62)$ & $92.31( \pm 11.12)$ & 0.45 \\
\hline Total Score & $183.84( \pm 20.83)$ & $174.58( \pm 28.18)$ & 0.04 \\
\hline
\end{tabular}

Table 7: Complications

\begin{tabular}{|l|c|c|}
\hline & MTRO Cohort & Control Cohort \\
\hline Manipulation & 2 & 1 \\
\hline DVT/PE & 1 & 1 \\
\hline Blood transfusion & 0 & 1 \\
\hline Total & 3 & 3 \\
\hline
\end{tabular}

significant increase in the total function $(\mathrm{p}=0.04)$ and total knee score in the MTRO cohort ( $\mathrm{p}=0.04$; Table 6$)$.

\section{Complications/Revisions}

The overall complication rate was similar and limited in both cohorts (4\%). The most common complication was a closed manipulation for arthrofibrosis (Table 7).

We identified one revision surgery in a 66 year old female, with a preoperative BMI of 39.0 and no other significant medical comorbidities, six years postoperatively. The patient was noted to have increasing medial tibial resorption and then collapse as well as aseptic tibial loosening. This patient was revised to a stemmed tibial component two years postoperatively with no additional loosening at final follow-up.

\section{Discussion}

Numerous studies have evaluated methods for obtaining precise coronal alignment in the varus knee $[\underline{1}, \underline{5}]$. Recently, the MTRO has been described as an alternative or additive procedure to improve the correction achieved in arthritic knees with severe fixed varus deformities [ $[\underline{8}, \underline{8}]$. Dixon et. al. performed an MTRO on 10 patients with substantial varus deformity (mean 24 degrees). They noted improvements in Knee Society and function scores (24 and 34 to 94 and 85) with no patient requiring revision surgery. The average follow-up tibiofemoral angle was 4 degrees. More recently, Mullaji and Shetty evaluated 71 primary TKAs in patients that required an MTRO [12]. They specifically wanted to determine the amount of deformity correction achieved with an MTRO and correlate the amount of tibia osteotomized with the amount of correction achieved. For every $2 \mathrm{~mm}$ of tibial bone resected, they achieved one degree of deformity correction.

We performed a retrospective review of patients with varus deformities requiring a TKA to determine the patient and radiographic factors that are associated with a need to perform a MTRO, to review the radiographic and clinical outcomes of patients that underwent an MTRO, and lastly to compare the results of patients that required an MTRO to a matched cohort of TKA patients with a varus deformity that did not require a MTRO. At our institution, the senior author proceeds with a systematic approach for achieving coronal alignment. The MTRO is utilized for severe fixed varus deformities that are uncorrectable with sequential medial soft tissue releases.

We noted several limitations to our study. First, this was a retrospective review and therefore suffers all of the limitations of this type of study. Secondly, we have relatively short-term follow-up duration. However, we did not believe longer follow-up was necessary to show the ability of the MTRO to safely and accurately achieve coronal alignment. Thirdly, the radiographic measurements obtained from this study were measured utilizing standing AP knee radiographs (14" X 17" films) as opposed to full-length standing films. The AP knee radiographs have been shown to have no statistically significant difference in measuring alignment in patients compared with full-length standingfilms in previous studies [13]. Additionally, we only routinely obtain full length standing films at the six week postoperative evaluation. Therefore, we elected to review the AP knee radiographs at all time periods to standardize the measurements throughout the duration of the study. Next, we did not compare similar cohorts with regards to preoperative varus deformity. However, we note that preoperative deformity is associated with requiring an MTRO and therefore we were unable to match the cohort by this variable. Finally, we performed a matched cohort for age, gender, and BMI to control for patient demographic data that may affect outcomes. However, there may be several other patient comorbidities that could impact outcomes that 
were not controlled.

Utilizing a systematic approach for obtaining coronal balance in the varus knee potentially avoids the need to release the superficial MCL. Preoperatively, there was a statistically significant increase in varus tibiofemoral angles in the MTRO cohort. Although there was a statistical difference in alignment between the two groups, there was no clinically significant difference in the final postoperative tibiofemoral alignment. We were able to obtain alignment within approximately one degree of the control cohort. Additionally, no patient in the MTRO group required implantation of a constrained implant or required a superficial MCL release. We believe that the MTRO offers a safe and reliable modality for achieving a well-balanced TKA, while avoiding the potential risks of injury to the superficial MCL that can occur with both traditional and piecrusting techniques of release [14].

The pre-operative tibiofemoral and tibial articular surface angles were noted to be statistically in more varus alignment in the MTRO cohort. The majority of the varus deformity seen in varus gonarthrosis was derived from the tibia, with minimal contribution from the femoral anatomy. The femoral articular surface angle was not significantly different between the cohorts. Additionally, we did not identify any patient-specific risk factors including weight, age, or gender. The pre-operative tibiofemoral deformity identified on pre-operative radiographs is likely the best predictor for patients that will require an MTRO.

Both cohorts exhibited excellent clinical results with mean Knee Society total knee scores of 183.84 and 174.58 for the MTRO and the control cohorts, respectively. The combined and function Knee Society scores were statistically better in the MTRO group but likely do not represent substantial clinical differences. Additionally, both groups demonstrated similar range of motion and pain scores. The revision rates and complication rates were low and similar between these groups showing the MTRO can be performed without increased risk at short- to mid-term followup evaluation.

An interesting post-operative radiographic finding was the presence of medial tibial bone resorption underneath the medial periphery of the tibial baseplate, observed in $64 \%$ of the MTRO patients (Figures 6A and 6B). Only two patients in the control cohort were noted to have medial tibial bone resorption ( $p=0.008$ ). Initially, we speculated that the presence of medial tibial bone resorption was due to thermal necrosis from the saw blade used to trim the sclerotic medial tibia during an MTRO. However, it also could be related to stress shielding as we noted a statistically significant increase in medial tibial bone resorption in patients with a metal backed rotating platform tibial tray

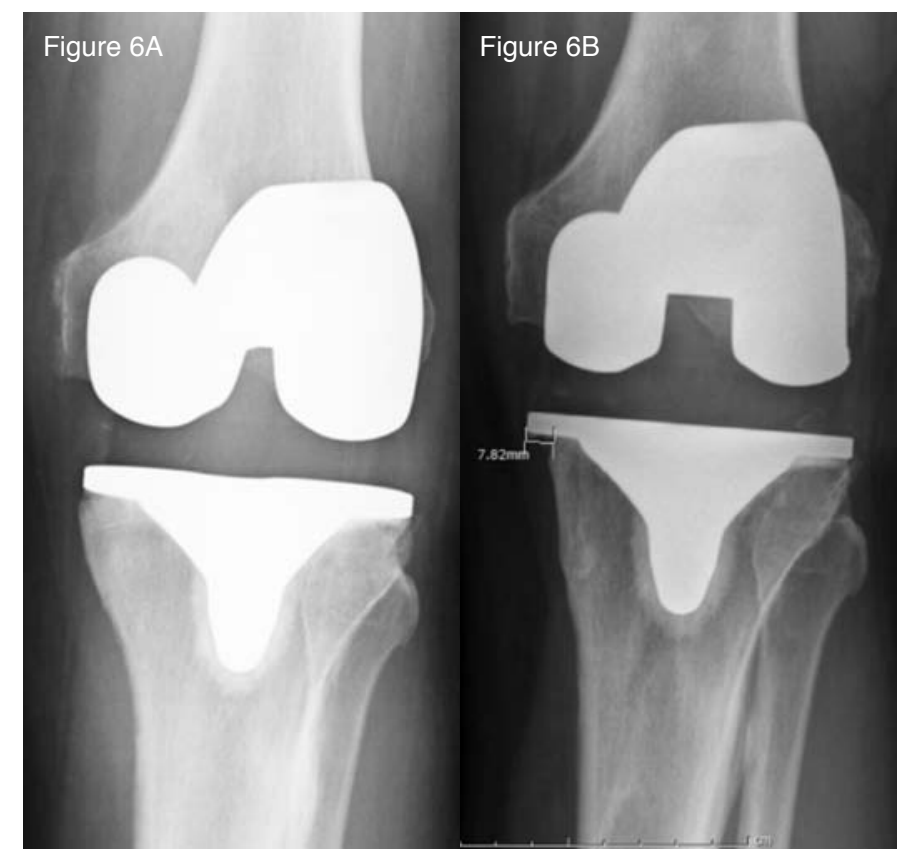

Figure 6A \& B: Radiographic finding of the presence of medial tibial bone resorption underneath the medial periphery of the tibial baseplate.

compared to an all polyethylene tibial component (37/48 $(77 \%)$ vs $1 / 12(8 \%), \mathrm{p}<0.01)$. This has led the authors to speculate that the medial tibial bone resorption may be a function of stress shielding in combination with potential thermal necrosis. The mechanism may be related to unloading the medial tibial plateau by correcting the varus alignment and further stress-shielding by utilizing a cobalt chromium tibial base plate. Additionally, the two patients that had medial tibial bone resorption in the control cohort were implanted with a cobalt chromium tray. Further investigation is required to determine the exact etiology of this unique phenomenon. At mid-term follow-up duration, this does not appear to be clinically significant, however, close monitoring of these patients will continue at our institution. In light of this observation, the authors now perform the MTRO with a rongeur or add irrigation if the sclerotic medial tibial is being removed using a saw to lessen the potential for thermal necrosis.

\section{Conclusion}

A MTRO was found to be a safe and effective method of achieving coronal balance and alignment in patients with fixed varus knee deformity requiring a TKA. No patient in the MTRO group required a release of the superficial MCL or required increased implant constraint. The MTRO was associated with statistically significantly improved Knee Society scores and similar final range of motion. Additionally, there was no substantial difference in revision 
or complication rates between the MTRO and control cohorts. Medial tibial bone resorption was present in over half of the patients in which a MTRO was performed. The exact mechanism of this phenomenon is unknown at this time but may be related to either stress shielding or thermal necrosis associated with the osteotomy. Longer follow-up is necessary to determine if this finding will result in any adverse clinical outcomes. However, at this time, the medial tibial bone resorption did not impact implant survivorship or patient outcomes and requires continued surveillance. We currently recommend the use of an MTRO to achieve coronal balance in patients with substantial varus deformity.

\section{Disclosure}

The authors declare that there is no conflict of interest regarding the publication of this paper. For full disclosures refer to last page of this journal.

\section{References}

1. Whiteside LA. Soft tissue balancing: the knee. J Arthroplasty. 2002;17(4 Suppl 1):23-27. http://www.ncbi.nlm.nih.gov/pubmed/12068398. Accessed August 9, 2015.

2. Teeny SM, Krackow KA, Hungerford DS, Jones M. Primary total knee arthroplasty in patients with severe varus deformity. A comparative study. Clin Orthop Relat Res. 1991;(273):19-31. http://www.ncbi.nlm.nih.gov/pubmed/1959270. Accessed August 9, 2015.

3. Matsumoto T, Muratsu H, Kubo S, Matsushita T, Kurosaka M, Kuroda R. The influence of preoperative deformity on intraoperative soft tissue balance in posterior-stabilized total knee arthroplasty. J Arthroplasty. 2011;26(8):1291-1298. doi:10.1016/j.arth.2011.01.003.
4. De Muylder J, Victor J, Cornu O, Kaminski L, Thienpont E. Total knee arthroplasty in patients with substantial deformities using primary knee components. Knee Surg Sports Traumatol Arthrosc. September 2014. doi:10.1007/s00167-0143269-X.

5. Seo J-G, Moon Y-W, Jo B-C, Kim Y-T, Park S-H. Soft Tissue Balancing of Varus Arthritic Knee in Minimally Invasive Surgery Total Knee Arthroplasty: Comparison between Posterior Oblique Ligament Release and Superficial MCL Release. Knee Surg Relat Res. 2013;25(2):60-64. doi:10.5792/ksrr.2013.25.2.60.

6. Meloni MC, Hoedemaeker RW, Violante B, Mazzola C. Soft tissue balancing in total knee arthroplasty. Joints. 2(1):37-40. http://www.pubmedcentral.nih.gov/articlerender.fcgi?artid $=4295665 \&$ tool=pmcentrez\&rendertype $=$ abstract.. Accessed August 9, 2015.

7. Dixon MC, Parsch D, Brown RR, Scott RD. The correction of severe varus deformity in total knee arthroplasty by tibial component downsizing and resection of uncapped proximal medial bone. J Arthroplasty. 2004;19(1):19-22. http://www. ncbi.nlm.nih.gov/pubmed/14716645. Accessed August 9, 2015.

8. Krackow KA, Raju S, Puttaswamy MK. Medial over-resection of the tibia in total knee arthroplasty for varus deformity using computer navigation. J Arthroplasty. 2015;30(5):766-769. doi:10.1016/j.arth.2014.11.034.

9. Niki Y, Harato K, Nagai K, Suda Y, Nakamura M, Matsumoto M. Effects of Reduction Osteotomy on Gap Balancing During Total Knee Arthroplasty for Severe Varus Deformity. J Arthroplasty. July 2015. doi:10.1016/j.arth.2015.06.061.

10. Ahn JH, Back YW. Comparative Study of Two Techniques for Ligament Balancing in Total Knee Arthroplasty for Severe Varus Knee: Medial Soft Tissue Release vs. Bony Resection of Proximal Medial Tibia. Knee Surg Relat Res. 2013;25(1):13-18. doi:10.5792/ksrr.2013.25.1.13.

11. Matsueda M, Gengerke TR, Murphy M, Lew WD, Gustilo RB. Soft tissue release in total knee arthroplasty. Cadaver study using knees without deformities. Clin Orthop Relat Res. 1999;(366):264-273. http://www.ncbi.nlm.nih.gov/ pubmed/10627744. Accessed August 9, 2015.

12. Mullaji AB, Shetty GM. Correction of varus deformity during TKA with reduction osteotomy. Clin Orthop Relat Res. 2014;472(1):126-132. doi:10.1007/s11999013-3077-5.

13. Skyttä ET, Haapamäki V, Koivikko M, Huhtala H, Remes V. Reliability of the hip-to-ankle radiograph in determining the knee and implant alignment after total knee arthroplasty. Acta Orthop Belg. 2011;77(3):329-335. http://www.ncbi.nlm. nih.gov/pubmed/21846000. Accessed September 29, 2015.

14. Meneghini RM, Daluga AT, Sturgis LA, Lieberman JR. Is the pie-crusting technique safe for MCL release in varus deformity correction in total knee arthroplasty? J Arthroplasty. 2013;28(8):1306-1309. doi:10.1016/j.arth.2013.04.002. 